\title{
Evaluation of an orthotopically implanted calcium phosphate cement containing gelatin microparticles
}

\author{
Dennis P. Link, ${ }^{1}$ Juliette van den Dolder, ${ }^{1}$ Jeroen J. J. P. van den Beucken, ${ }^{1}$ Wouter Habraken, ${ }^{1}$ \\ Annemieke Soede, ${ }^{2}$ Otto C. Boerman, ${ }^{2}$ Antonios G. Mikos, ${ }^{3}$ John A. Jansen ${ }^{1}$ \\ ${ }^{1}$ Department of Periodontology and Biomaterials, Radboud University Nijmegen Medical Center, \\ Nijmegen, The Netherlands \\ ${ }^{2}$ Department of Nuclear Medicine, Radboud University Nijmegen Medical Center, Nijmegen, The Netherlands \\ ${ }^{3}$ Department of Bioengineering, Rice University, Houston, Texas, USA
}

Received 22 December 2007; revised 4 March 2008; accepted 27 March 2008

Published online 2 June 2008 in Wiley InterScience (www.interscience.wiley.com). DOI: 10.1002/jbm.a.32091

\begin{abstract}
This study focused on the degradation properties of gelatin microparticles incorporated in calcium phosphate $(\mathrm{CaP})$ cement and the subsequent effect of these composites on bone formation. Positively charged alkaline gelatin (type A) microparticles or negatively charged acidic gelatin (type B) microparticles were incorporated in $\mathrm{CaP}$ cement, which was implanted in critical-sized cranial defect in rats and left in place for 2, 4, and 8 weeks. The degradation of the gelatin was monitored using radioiodinated microparticles. After 4 and 8 weeks of implantation, a significantly faster degradation of type A gelatin over type B gelatin was found. Light microscopic analysis of the specimens showed similar bone response concerning implants containing either type A or B gelatin microparticles. At 2 weeks of implantation, a minimal amount of bone formation was observed from the cranial bone toward the implant, while after 8 weeks of implantation an
\end{abstract}

entire layer of newly formed bone was present from the cranial bone toward the implant periphery. Bone ingrowth into the implant was observed at sites of gelatin microparticle degradation, predominantly at the implant periphery. Histomorphometrical evaluation did not reveal significant differences in bone formation between $\mathrm{CaP}$ cement incorporated with either type A or B gelatin microparticles during implantation periods up to 8 weeks. In conclusion, this study demonstrates that gelatin type influences the degradation of gelatin microparticles incorporated in $\mathrm{CaP}$ cements. However, this difference in degradation and the concomitant subsequent macroporosity did not induce differences in the biological response. (C) 2008 Wiley Periodicals, Inc. J Biomed Mater Res 90A: 372-379, 2009

Key words: CaP cement; gelatin microparticles; degradation; bone ingrowth; critical-sized defect

\section{INTRODUCTION}

Porous calcium phosphate $(\mathrm{CaP})$ cements are promising scaffold materials in bone tissue-engineering approaches. These $\mathrm{CaP}$ cements consist of a mixture of powder and liquid, which hardens in situ as a result of the entanglement of newly formed crystals at body temperature. Although injectable CaP cement shows excellent biocompatibility and osteoconductivity, resorption in vivo is slow. ${ }^{1}$ The intrinsic nanoporosity of the $\mathrm{CaP}$ cement only allows transport of nutrients and waste and does not allow tissue ingrowth. ${ }^{2-4}$ Therefore, methods have been

Correspondence to: J. A. Jansen; e-mail: j.jansen@dent. umcn.nl

Contract grant sponsor: Ministry of Economic Affairs; contract grant number: NGT6205

(C) 2008 Wiley Periodicals, Inc. developed to accelerate resorption and tissue ingrowth by creating microporosity in the $\mathrm{CaP}$ cement. One method involves the incorporation of polymeric microparticles, such as poly(D,L-lactic-coglycolic acid) (PLGA) microparticles inside the CaP cement. ${ }^{3,5-8}$ Previous studies showed that the degradation of PLGA microparticles takes 6-12 weeks. $^{5}$ This slow degradation is likely to prevent rapid cell penetration into the implant, which would delay intraimplant bone formation. Even more ideally, a scaffold that contains microparticles with variation in degradation rates is preferred, hence allowing the creation of bone substitute materials, which combine rapid cell penetration (after rapid microparticle degradation) with a sustained release of appropriate growth factors (from slow degrading microparticles).

For microparticle preparation, the use of a polymer from natural sources, such as gelatin, is appealing as a biodegradable, biocompatible, and nontoxic substitute for synthetic degradable polymers. ${ }^{9,10}$ 
During the fabrication process of commercially available gelatin either a positively charged alkaline gelatin (type A) or a negatively charged acidic gelatin (type B) at physiological $\mathrm{pH}$ can be obtained. ${ }^{11}$ The application of these types of gelatine for microparticle preparation further allows a certain degree of control over thermal and mechanical stability of the microparticles under physiological conditions and the related degradation rate by crosslinking with glutaraldehyde. ${ }^{12,13}$ After degradation of the gelatin microparticles by members of the matrix metalloprotease (MMP) family (including gelatinases and collagenases) ${ }^{14}$ microporosity is created in the $\mathrm{CaP}$ cement.

In view of the above mentioned, this study focused on the degradation properties and the subsequent effect on bone formation of gelatin microparticles incorporated in $\mathrm{CaP}$ cement in a critical-sized cranial defect in rats. $\mathrm{CaP} /$ gelatin composites were prepared using either alkaline or acidic gelatin microparticles. Degradation properties were obtained by measuring $\gamma$-emission of remaining radioiodinated gelatin microparticles in the $\mathrm{CaP}$ cement implants after implantation periods of 2, 4, and 8 weeks. Additionally, histological and histomorphometrical analyses were performed to evaluate general tissue responses and the amount of new bone formation.

\section{MATERIALS AND METHODS}

\section{Gelatin microparticles}

The preparation of gelatin microparticles was based on procedures described previously by Holland et al. ${ }^{15}$ Briefly, a gelatin solution was prepared by dissolving $2.5 \mathrm{~g}$ type A $(\mathrm{pI}=7.0-9.0)$ or type $\mathrm{B}(\mathrm{pI}=4.7-5.2)$ gelatin (Sigma, St. Louis, MO) in $22.5 \mathrm{~mL}$ MilliQ at $37^{\circ} \mathrm{C}$ for $1 \mathrm{~h}$. Then, this solution was added drop by drop to $125 \mathrm{~mL}$ chilled $\left(4^{\circ} \mathrm{C}\right)$ olive oil (Acros Organics, Geel, Belgium) under continuous stirring at $500 \mathrm{rpm}$. After $30 \mathrm{~min}, 50 \mathrm{~mL}$ chilled acetone $\left(4^{\circ} \mathrm{C}\right.$; HPLC grade, Labscan, Dublin, Ireland) together with $1 \mathrm{~mL} 25 \mathrm{w} / \mathrm{v} \%$ glutaraldehyde (Merck, Darmstadt, Germany) was added to the emulsion. After stirring for an additional $30 \mathrm{~min}$, the microparticles were collected by filtration and washed with chilled acetone $\left(4^{\circ} \mathrm{C}\right)$. Finally, the microparticles were frozen and lyophilized. Microparticle size distribution was assessed by morphometrical analysis using a light microscope (Leica Microsystems AG, Wetzlar, Germany) and computer-based image analysis techniques (Leica ${ }^{\circledR}$ Qwin Pro-Image Analysis System, Wetzlar, Germany).

\section{Radioiodization of gelatin microparticles}

Type A and B gelatin microparticles were labeled with Iodine $^{125}$ (Amersham Biosciences, Buckinghamshire, UK) using iodogen, a solid-phase oxidizing agent that catalyzes the reaction to achieve a chemical link between radiolabel and a protein. ${ }^{16}$ Briefly, $100 \mu \mathrm{L}$ of gelatin microparticles suspension in PBS $(100 \mathrm{mg} / \mathrm{mL})$ was reacted with $100 \mu \mathrm{Ci}$ Iodine $^{125}$ in a reaction vial coated with $50 \mu \mathrm{g}$ iodogen during $10 \mathrm{~min}$ at room temperature in $50 \mathrm{mM}$ phosphate buffer ( $\mathrm{pH}$ 7.4). The suspension was then centrifuged in an Eppendorf microcentrifuge (5000 rpm, $5 \mathrm{~min}$ ), and the pellet was washed four times with $1 \mathrm{~mL}$ PBS. The specific activity of the acidic and alkaline gelatin microparticles was $2 \mu \mathrm{Ci} / \mu \mathrm{g}$. Hot/cold suspensions of gelatin microparticles were prepared in ratio of 1:4 for the in vivo study.

\section{CaP/gelatin composites}

The CaP cement Calcibon ${ }^{\circledR}$ (Merck Biomaterial GmbH, Darmstadt, Germany) was used for the preparation of the $\mathrm{CaP} /$ gelatin composites. The chemical composition of this cement is $61 \% \alpha-\mathrm{TCP}, 26 \% \mathrm{CaHPO}_{4}, 10 \% \mathrm{CaCO}_{3}$, and $3 \%$ PHA $(\alpha-$ TCP is alpha tri-calcium phosphate, PHA is precipitated hydroxyapatite). Type A or B gelatin microparticles $(50 \mathrm{mg}$ ) were preswollen in $300 \mu \mathrm{L}$ water (complete absorption) and mixed for $15 \mathrm{~s}$ in a $2 \mathrm{~mL}$ syringe (Sherwood medical monoject) with closed tip using a mixing apparatus (Silamat, Vivadent, Schaan, Liechtenstein). CaP cement powder (950 mg) was added afterward in the $2 \mathrm{~mL}$ syringe. The final injectable composite was obtained by adding $350 \mu \mathrm{L}$ of $\mathrm{Na}_{2} \mathrm{HPO}_{4}(1 \mathrm{wt} \%)$ to $1000 \mathrm{mg}$ of this $\mathrm{CaP} /$ gelatin mixture and mixing it for $15 \mathrm{~s}$ with the mixing apparatus. Subsequently, the $\mathrm{CaP} /$ gelatin composite was immediately injected into teflon molds $(8.0 \mathrm{~mm}$ in diameter and $2.4 \mathrm{~mm}$ in height) to ensure a standardized shape of the composite discs. The composite discs were removed from these molds after cement setting at room temperature for 1 day. The total porosity of the different $\mathrm{CaP} /$ gelatin composites was determined by correlating the weight of $\mathrm{CaP}$ discs with the weight of $\mathrm{CaP} /$ gelatin discs after placement of the samples in a furnace at $650^{\circ} \mathrm{C}$ for $2 \mathrm{~h}$ to remove the gelatin microparticles. ${ }^{5}$

\section{Surgery}

Forty-eight Wistar male rats (weight $\pm 250 \mathrm{~g}$ ) were used, which individually received one CaP/gelatin composite disc implanted into a circular critical-sized cranial defect of $8 \mathrm{~mm} .{ }^{17}$ Half of the rats received discs with type A gelatin microparticles (18 without, 6 with radiolabel), and the other half received discs incorporated with type B gelatin microparticles (18 without, 6 with radiolabel), which were implanted for 2, 4, and 8 weeks. The animal experiment was approved by the Animal Ethical Committee of the Radboud University Nijmegen Medical Center and national guidelines for the care and use of laboratory animals were observed.

Surgery was performed under general inhalation anesthesia, induced by $4 \%$ isoflurane and maintained with $2.5 \%$ isoflurane by a nonrebreather mask. The rats were monitored with an oxy-pulse meter during surgery. To minimize postoperative pain, Fentanyl $^{\mathbb{B}}(3 \mathrm{~mL} / \mathrm{kg}$ i.p. $)$ 
was administered preoperatively and buprenorfine (Temgesic $^{\circledR}(0.05 \mathrm{mg} / \mathrm{kg}$ s.c.) postoperatively.

After anesthesia, the rats were immobilized on their abdomen, and the skull was washed and disinfected with iodine. A longitudinal incision was made down to the periosteum from the nasal bone to the occipital protuberance, and soft tissues were sharp dissected to visualize the cranial periosteum. Subsequently, a midline incision was made in the periosteum, and the periosteum was undermined and lifted off the parietal skull. To minimize pain, lidocaine was administered onto the periosteum before incision. To create a central full thickness bone defect in the parietal cranium, a hollow trephine drill (ACE dental implant systems, Portugal) with an outer diameter of $8.0 \mathrm{~mm}$ in a dental handpiece was used. The bone defect was carefully drilled under continuous cooling with physiologic saline, without damaging of the underlying dura. Then, the created bone segment was carefully removed, without damaging the underlying sagittal sinus. Following insertion of a CaP/gelatin composite disc, the periosteum was closed using resorbable Vicryl ${ }^{\mathbb{R}}$ 5-0 suture material. Subsequently, the skin was closed using resorbable Vicryl 4-0 sutures. To minimize postoperative discomfort, treatment with Temgesic ${ }^{\circledR}$ was continued for 2 days postoperatively. Two animals were housed in each cage. The proper intake of fluids and food was monitored in the first 5 days postoperatively. Also, the animals were observed for signs of pain, infection, and proper activity. The rats were euthanized 2, 4, or 8 weeks after surgery by an overdose of $\mathrm{CO}_{2} / \mathrm{O}_{2}$ suffocation.

\section{Degradation assay}

Before surgery, CaP discs incorporated with either type A or B radioiodinated gelatin microparticles (both $n=6$ ) were measured in a gamma well-type counter (Wizard, Pharmacia, Uppsala, Sweden). These samples were destined for implantation in a critical-sized cranial defect as mentioned earlier. After both 4 and 8 weeks of implantation, six rats (three with gelatin A and three with gelatin B) with the radioiodinated implants were euthanized by $\mathrm{CO}_{2}$ suffocation. The implants with their surrounding tissue were retrieved and measured in a gamma counter ex vivo. Standards were counted simultaneously to correct for radioactive decay. The remaining activity in the discs was expressed as percentage of the initial activity and is a criterion for the remaining amount of gelatin left in the composites.

\section{Scanning electron microscopy}

To obtain information about the morphological appearance of the composites, the 12 implants of the degradation assay were fixated in $10 \%$ formalin solution and dehydrated in a graded series of ethanol. After longitudinal breakage of the implants, samples were sputter-coated with gold and examined with SEM (Jeol 6310 scanning electron microscope, Boston, MA). SEM was performed at the Microscopic Imaging Center (MIC) of the Nijmegen
Center for Molecular Life Sciences (NCMLS), the Netherlands.

\section{Histological and histomorphometrical evaluation}

After euthanizing the rats, the implants with their surrounding tissue were retrieved and prepared for histological evaluation. The samples were fixated in $10 \%$ formalin solution $(\mathrm{pH}=7.4)$, dehydrated in a graded series of ethanol, and embedded in methylmethacrylate. Following polymerization, three $10-\mu \mathrm{m}$ thick sections were prepared per implant using a sawing microtome technique. ${ }^{8}$ The sections were stained with methylene blue and basic fuchsin and investigated with a light microscope. For the histomorphometrical analysis, all sections per implant were evaluated using a computer-based image analysis technique (Leica Qwin Pro-image analysis system, Wetzlar, Germany). The quantitative evaluation of newly formed bone was calculated by determining a region of interest (ROI), which was set between the surface area of both ends of the originally created critical-sized defect. Within this ROI, new bone formation was distinguished from preexisting bone and composite through structure and color differences. New bone formation was expressed in millimeter square, using standardization of the two-dimensional ROI.

\section{Statistical analysis}

Statistical analysis was performed with GraphPad ${ }^{\mathbb{R}}$ Instat 3.05 software (GraphPad Software, San Diego, CA) using analyses of variance (ANOVA) combined with a Tukey's test to correct for multiple comparison. Significance was set at $p<0.05$.

\section{RESULTS}

\section{Implant characterization}

The generation of type A gelatin microparticles resulted in a diameter distribution of 1-161 $\mu \mathrm{m}$ with an average size of $37.4 \pm 31.1 \mu \mathrm{m}$. The diameter distribution of the type $B$ gelatin microparticles varied between 1 and $66 \mu \mathrm{m}$ with an average size of $20.7 \pm$ $14.6 \mu \mathrm{m}$. The porosity of the implants was $48.7 \% \pm$ $0.1 \%$ for the implants containing type A gelatin microparticles and $45.0 \% \pm 1.3 \%$ for the implants containing type B gelatin microparticles (Table I).

\section{In vivo experiment}

\section{General observations}

All 48 Wistar male rats remained in good health and did not show any wound complications. At retrieval, the cranial implants were all covered by peri- 
TABLE I

Implant Macroporosity and Gelatin Microparticle Sizes

\begin{tabular}{lcc}
\hline & Cap/Gelatin A & Cap/Gelatin B \\
\hline Macroporosity & $48.7 \%$ & $45.0 \%$ \\
Microparticles size & $15.6 \pm 13.8 \mu \mathrm{m}$ & $8.4 \pm 7.6 \mu \mathrm{m}$ \\
(before swelling) & $(1$ to $63 \mu \mathrm{m})$ & $(1$ to $49 \mu \mathrm{m})$ \\
Microparticle size & $37.4 \pm 31.1 \mu \mathrm{m}$ & $20.7 \pm 14.6 \mu \mathrm{m}$ \\
(after swelling) & $(1$ to $161 \mu \mathrm{m})$ & $(1$ to $66 \mu \mathrm{m})$ \\
\hline
\end{tabular}

osteum and implants remained intact. No inflammatory signs or adverse tissue reactions were observed.

\section{SEM examination}

SEM examination of CaP/gelatin composites after implantation (Fig. 1) showed a homogeneous distribution of type $\mathrm{A}$ and $\mathrm{B}$ gelatin microparticles throughout the implants. The gelatin type A composites contained larger microparticles compared to gelatin type B composites. The 4- and 8-week implantation period showed a similar composite morphology. Generally, gelatin microparticle degradation appeared to start at the implant periphery, as microparticles in the core of the implant appeared to be intact. However, surface erosion of microparticles was observed within all samples, indicated by the deformation of microparticle structure [Fig. 1(E)]. No apparent differences were observed between implants containing either type A or B gelatin.

\section{Degradation study}

The implants with their surrounding tissue were retrieved, and the retained activity was determined
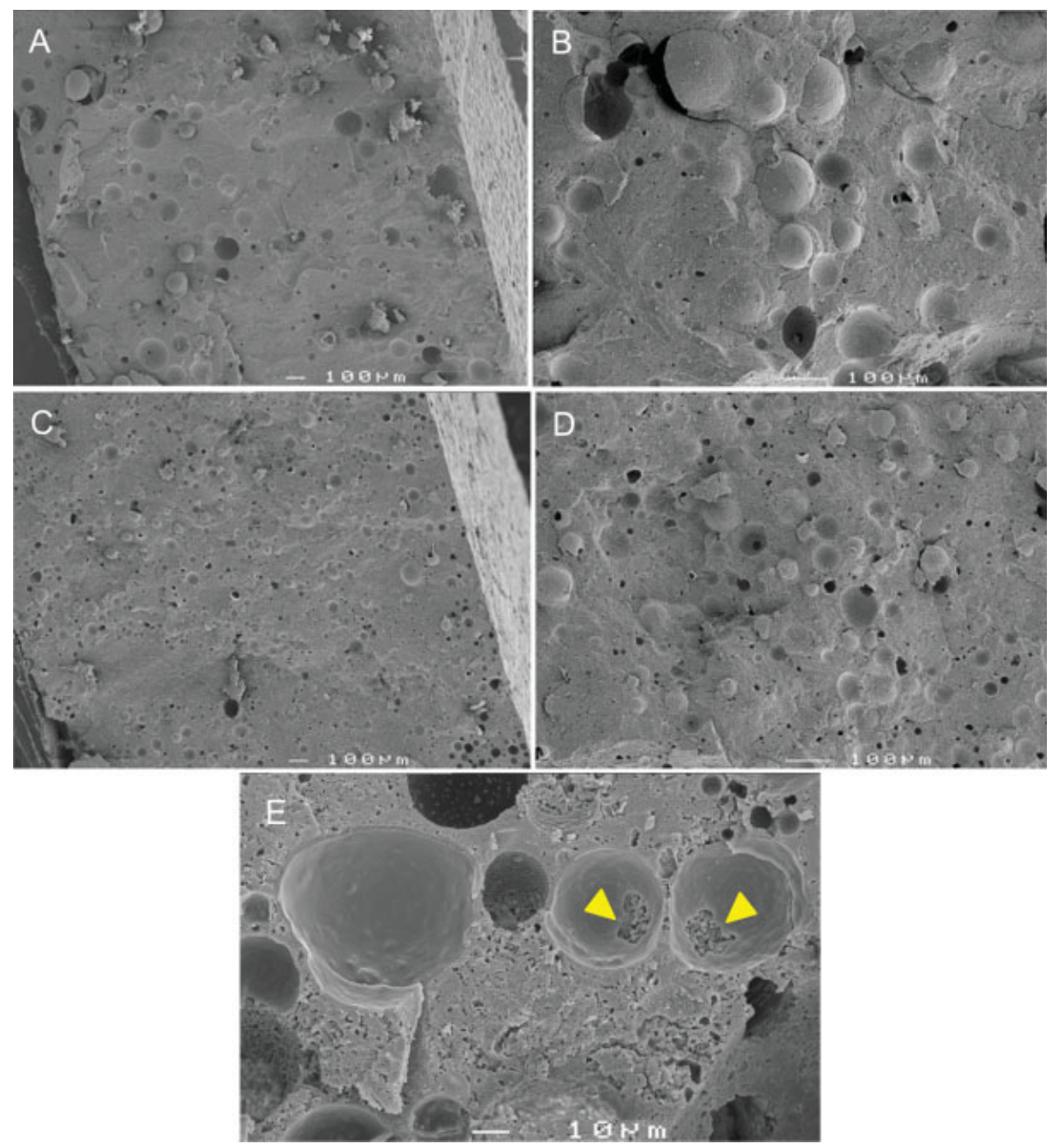

Figure 1. SEM examination of type $A(A, B)$ and B (C-E) gelatin microparticles incorporated in CaP cement implants after eight weeks of implantation. Surface erosion of microparticles is observed, indicated by the deformation of the microparticle structure (arrowheads). [Color figure can be viewed in the online issue, which is available at www.interscience.wiley. com.] 


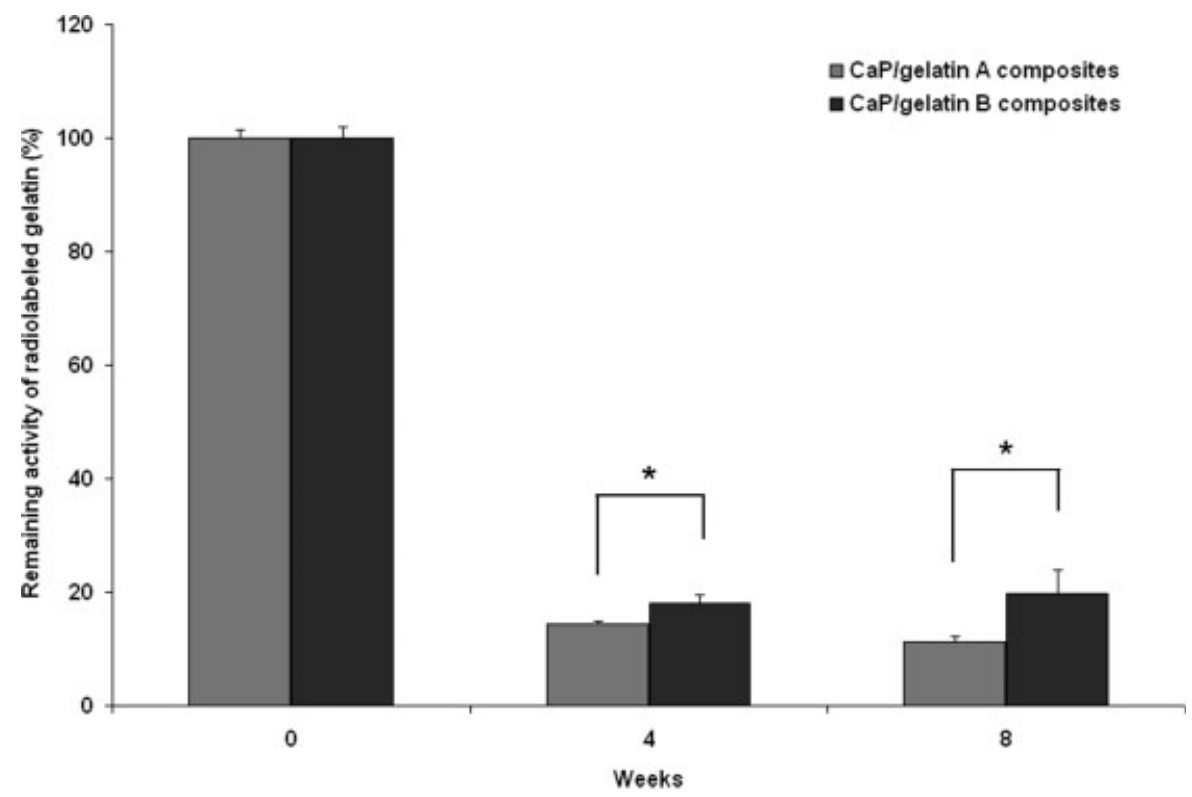

Figure 2. Remaining iodine125 activity (in \%) of the types A and B gelatin microparticles incorporated in CaP cement implants. Significant differences $(p<0.05)$ are indicated with an asterisk $\left.{ }^{*}\right)$.

using a gamma counter $e x$ vivo. The remaining activity of the radioiodinated type A gelatin microparticles was $14.3 \% \pm 0.5 \%$ and $11.3 \% \pm 0.9 \%$ after 4 and 8 weeks of implantation, respectively. Radioiodinated type B gelatin microparticles showed a remaining activity of $18.1 \% \pm 1.4 \%$ and $19.6 \% \pm 4.4 \%$ after 4 and 8 weeks of implantation, respectively. Statistical analysis showed significantly lower remaining activity for type A compared to type B gelatin microparticles after both 4 and 8 weeks of implantation (Fig. 2).

\section{Histological and histomorphometrical evaluation}

Light microscopic analysis of the histological sections of the implants showed similar results for implants containing either type A or B gelatin microparticles. The 2-week implantation period [Fig. 3 $(\mathrm{A}, \mathrm{D})]$ resulted in minimal bone formation from the cranial bone toward the implant. After 4 weeks of implantation [Fig. 3(B,E)], a thin layer of newly formed bone was present from the cranial bone
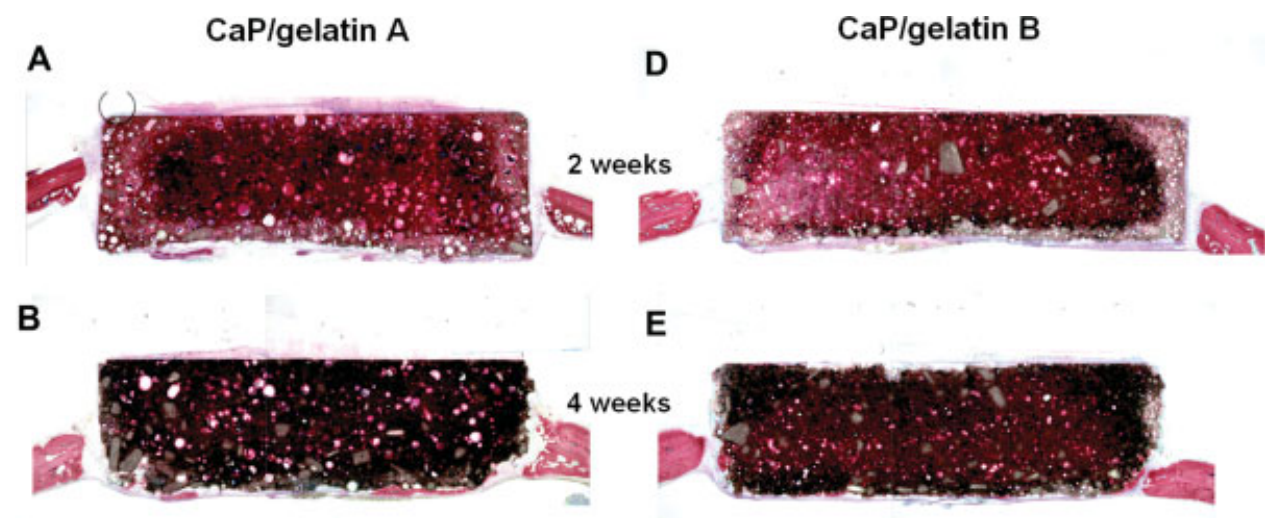

E
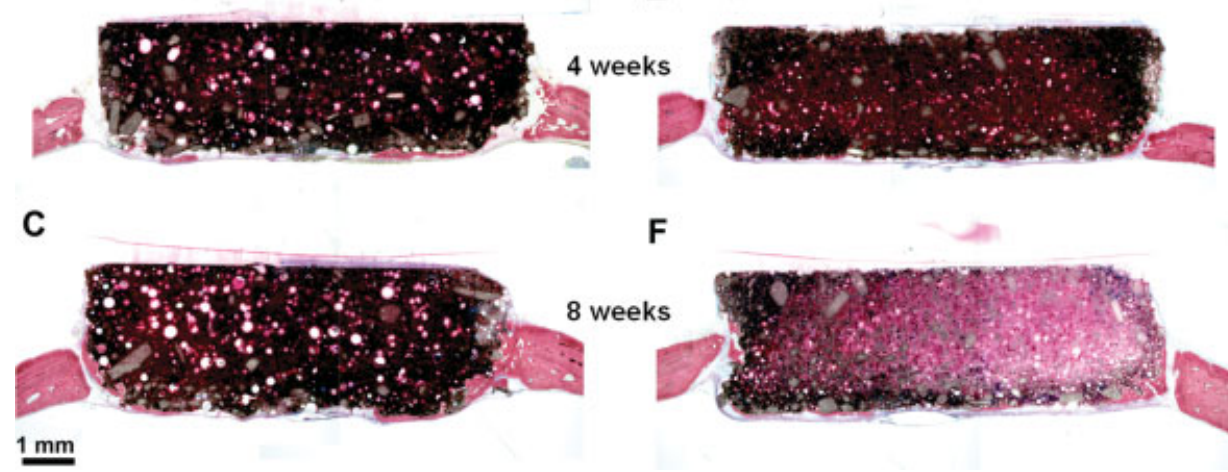

$\mathbf{F}$

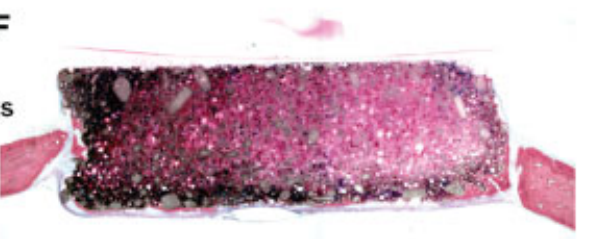

Figure 3. Histological sections of $\mathrm{CaP}$ cement implants containing type A gelatin microparticles after 2 (A), 4 (B), and 8 (C) weeks of implantation as well as implants containing type B gelatin microparticles after 2 (D), 4 (E), and 8 (F) weeks of implantation. [Color figure can be viewed in the online issue, which is available at www.interscience.wiley.com.] 

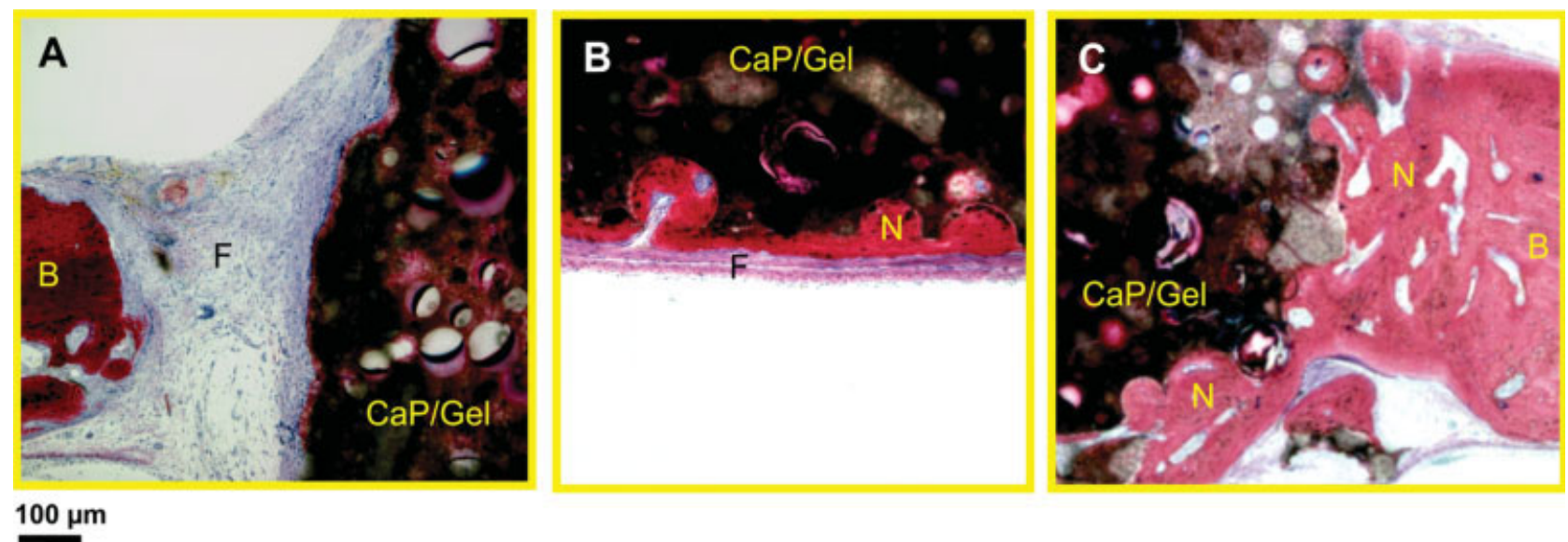

Figure 4. Magnification of histological sections of CaP cement implants containing type A gelatin microparticles after 2 (A) and 8 (B,C) weeks of implantation. (B, cranial bone; F, fibrous tissue; and N, newly formed bone). [Color figure can be viewed in the online issue, which is available at www.interscience.wiley.com.]

toward the implant periphery. Occasionally, limited bone formation at the cerebral side of the implant was observed. After 8 weeks of implantation [Fig. $3(\mathrm{C}, \mathrm{F})]$, a layer of newly formed bone was present from the cranial bone toward the implant periphery. Bone ingrowth into the implant was predominantly observed at sites of gelatin microparticles degradation at the implant periphery [Fig. 4(C)]. Also, bone formation at the cerebral side of the implants was observed, although no bridging of the entire defect was observed [Fig. 4(B)]. At all implantation periods, fibrous tissue between implants and cranial bone was observed, especially in case there was no close contact between implant and bone [Fig. 4(A)]. No bone or fibrous tissue was observed in the center of the implants after any of the implantation periods.

Histomorphometrical evaluation showed no significant differences between composites with either type A or B gelatin microparticles regarding bone formation after any of the implantation periods (Fig. 5). Generally, only limited amounts of newly formed bone were found in both type A and B gelatin microparticle composites. Implants containing type A microparticles showed $0.03 \pm 0.04 \mathrm{~mm}^{2}$ new bone after 2 weeks, $0.37 \pm 0.23 \mathrm{~mm}^{2}$ after four weeks, and finally $0.60 \pm 0.50 \mathrm{~mm}^{2}$ after 8 weeks of implantation. The samples containing type $\mathrm{B}$ gelatin microparticles showed $0.07 \pm 0.07 \mathrm{~mm}^{2}$ new bone after

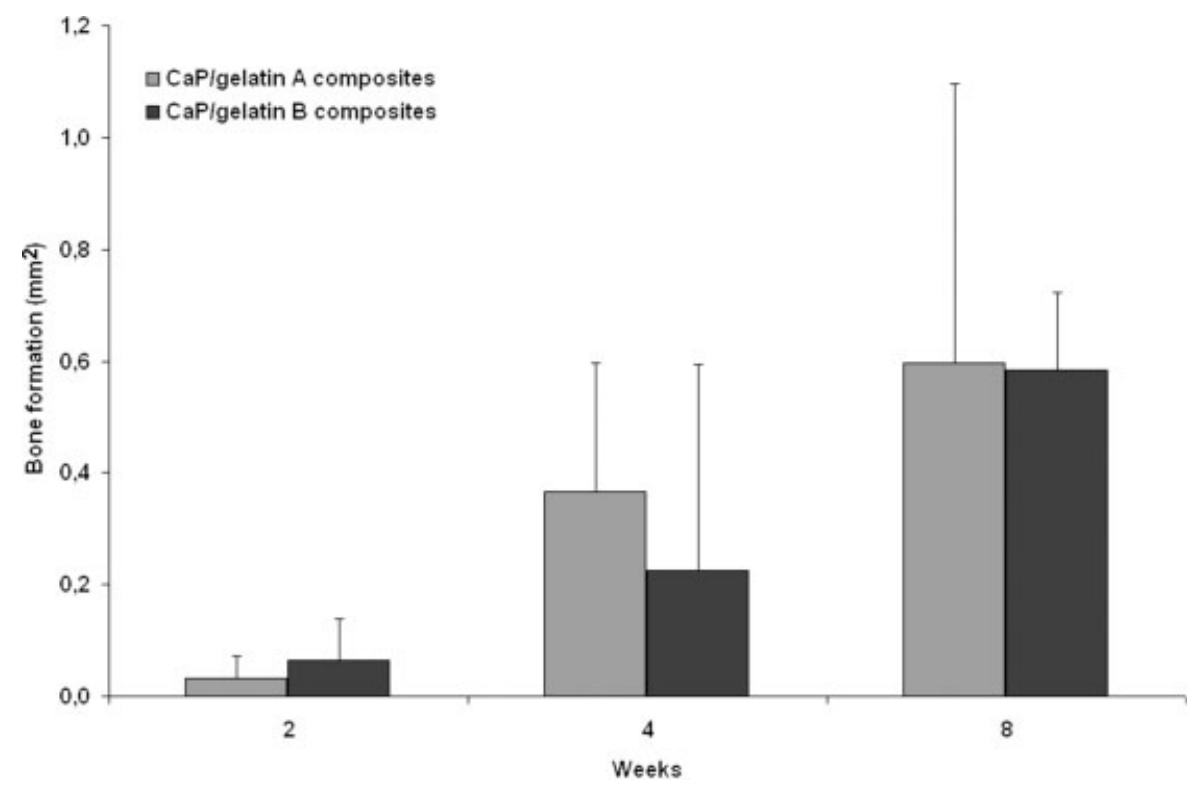

Figure 5. New bone formation (in $\mathrm{mm}^{2}$ ) in CaP implants containing either type A or B gelatin microparticles after 2, 4, and 8 weeks of implantation. 
2 weeks, $0.23 \pm 0.37 \mathrm{~mm}^{2}$ after 4 weeks, and finally $0.58 \pm 0.14 \mathrm{~mm}^{2}$ after 8 weeks of implantation.

\section{DISCUSSION}

In this study, the degradation properties and the subsequent effect on bone formation of gelatin microparticles incorporated in $\mathrm{CaP}$ cement in a critical-sized cranial defect in rats were examined. More specifically, the use of a natural polymeric material for the generation of microparticles was evaluated, because such a material is hypothesized to degrade more rapidly when compared with synthetic polymeric materials. The natural polymer gelatin was used in either an alkaline or an acidic form to reveal potential differences in degradation of resulting microparticles.

Implant characterization demonstrated that the macroporosity of the composites was $48.7 \% \pm 0.1 \%$ for composites containing type A gelatin microparticles and $45.0 \% \pm 1.3 \%$ for the composites containing type B microparticles. In view of previous studies with PLGA microparticles, ${ }^{8}$ this porosity should be high enough to obtain an interconnective network after degradation. However, porosity is not the only important factor in interconnectivity. Previous research showed that the interconnection was not solely relying on high porosity, but merely on microparticle size. ${ }^{8}$ Nonetheless, no fibrous tissue or bone formation was observed in this study throughout the implants, which is the parameter to indicate in vivo interconnectivity. Although no CaP precipitates were observed in this study, Habraken et al..$^{5}$ showed that $\mathrm{CaP}$ can precipitate inside and onto the gelatin microparticles. Consequently, after degradation of two adjacent microparticles, a shell of hydroxyapatite was created, instead of an interconnection. This could explain insufficiency of the used 5 wt \% gelatin microparticles in this study to obtain interconnected pores. Therefore, increasing the microparticle weight percentage is likely to solve this problem at least partially, although precipitation of $\mathrm{CaP}$ still can occur and problems with setting time of the composites could emerge if the weight percentage is above a certain threshold. ${ }^{17}$

SEM examination showed that gelatin microparticles were degraded at the implant periphery, whereas in the core of the implant, microparticles appeared to be intact. Before microparticle degradation, our implants only possessed an intrinsic nanoporosity without an interconnected microporous network. Therefore, fluid flow in the center of the implants was likely limited compared to that at the implant periphery. As a result, binding and/or activation of proteolytic enzymes can be different at these sites, thus limiting gelatin degradation in the center of the implants. ${ }^{5}$

The degradation study showed that the remaining radioactivity of the composites containing type A gelatin microparticles was lower than the composites containing type B gelatin microparticles. The difference between type A and B gelatin microparticles degradation was significant. This could be explained by a higher porosity and larger microparticle size of the type A gelatin, which results in faster degradation of the microparticles. ${ }^{17}$ Furthermore, the difference in charge and acidity could also account for the differences in degradation. Remarkably, the difference between the remaining radioactivity after 4 and 8 weeks implantation was minimal. Apparently, radioiodinated gelatin was still present in the core of the composites. This could be explained by the binding properties of gelatin to $\mathrm{CaP}^{18}$ and the high binding affinity of $\mathrm{CaP}$ cement for proteins, ${ }^{19,20}$ resulting in a strong bond between the $\mathrm{CaP}$ cement and the gelatin.

Unfortunately, long-term measurements with the same composites in vivo could not be performed, due to the low energetic emission of the Iodine ${ }^{125}$ label. Other radioactive isotopes (Iodine ${ }^{131}$ and Indium ${ }^{111}$ ) were considered for gelatin microparticle labeling, but were unsuitable to use for gamma camera measurements due to their short half-lives of 8 and 3 days, respectively. Therefore, samples could only be measured in a gamma counter ex vivo before implantation and after sacrificing the animals. Furthermore, for the chosen method to study gelatin microparticle degradation, the assumption was made that the chemical linkage of the radiolabel resulted in homogeneously radiolabeled microparticles rather than radiolabeling according to a gradient. This assumption is plausible because the microparticles are hollow and consist of a thin shell of gelatin. ${ }^{5}$ However, the authors recognize the limitations of this method. Therefore, it is likely that the radioactivity measurements represent an indirect method to determine gelatin microparticle degradation. It needs to be emphasized, however, that the measured radioactivity includes not only the remaining intact radioiodinated gelatin microparticles, but also remaining partially degraded gelatin microparticles. Therefore, the measured radioactivity does not automatically indicate that the microparticles are completely degraded.

Histological evaluation showed that fibrous tissue was observed between composite and cranial bone, especially in case there was no close contact between implant and bone. No bone or fibrous tissue was observed in the center of the composites after any of the implantation periods, probably due to the lack of interconnectivity. Therefore, bone ingrowth was only observed at the periphery of the composites after degradation of gelatin microparticles. 
Histomorphometrical evaluation showed that between implants containing type A and B gelatin microparticles, no significant differences in bone formation occurred during all implantation periods. This could be explained by the excellent osteoconductivity of the CaP cement. ${ }^{1,21,22}$ However, the amount of newly formed bone in this study was limited when compared with previous research with $\mathrm{CaP}$ cement implants. ${ }^{4,7,22}$ Factors including surgical technique, differences between animals or implant locations are known to effect bone formation. ${ }^{23}$

\section{CONCLUSIONS}

In conclusion, this study demonstrates that gelatin type influences the degradation of gelatin microparticles incorporated in $\mathrm{CaP}$ cements. However, this difference in degradation and the concomitant subsequent macroporosity did not induce differences in the biological response.

The authors thank the Dutch Technology Foundation (STW) applied science division of NWO.

\section{References}

1. Ooms EM, Wolke JG, van der Waerden JP, Jansen JA. Trabecular bone response to injectable calcium phosphate (Ca-P) cement. J Biomed Mater Res 2002;61:9-18.

2. del Real RP, Wolke JG, Vallet-Regi M, Jansen JA. A new method to produce macropores in calcium phosphate cements. Biomaterials 2002;23:3673-3680.

3. Ruhe PQ, Hedberg-Dirk EL, Padron NT, Spauwen PH, Jansen JA, Mikos AG. Porous poly(DL-lactic-co-glycolic acid)/calcium phosphate cement composite for reconstruction of bone defects. Tissue Eng 2006;12:789-800.

4. Ooms EM, Wolke JG, van de Heuvel MT, Jeschke B, Jansen JA. Histological evaluation of the bone response to calcium phosphate cement implanted in cortical bone. Biomaterials 2003;24:989-1000.

5. Habraken WJ, Wolke JG, Mikos AG, Jansen JA. Injectable PLGA microsphere/calcium phosphate cements: Physical properties and degradation characteristics. J Biomater Sci Polym Ed. 2006;17:1057-1074.

6. Simon CG Jr, Khatri CA, Wight SA, Wang FW. Preliminary report on the biocompatibility of a moldable, resorbable, composite bone graft consisting of calcium phosphate cement and poly(lactide-co-glycolide) microspheres. J Orthop Res 2002;20:473-482.

7. Link DP, van den Dolder J, Jurgens WJ, Wolke JG, Jansen JA. Mechanical evaluation of implanted calcium phosphate cement incorporated with PLGA microparticles. Biomaterials 2006;27:4941-4947.
8. Link DP, van den Dolder J, van den Beucken JJJP, Cuijpers VM, Wolke JGC, Mikos AG, Jansen JA. Evaluation of the biocompatibility of calcium phosphate cement/PLGA microparticle composites. J Biomed Mater Res A 2008;87:760-769.

9. Young S, Wong M, Tabata Y, Mikos AG. Gelatin as a delivery vehicle for the controlled release of bioactive molecules. J Control Release 2005;109:256-274.

10. Habraken WJ, Wolke JG, Jansen JA. Ceramic composites as matrices and scaffolds for drug delivery in tissue engineering. Adv Drug Deliv Rev 2007;59:234-248.

11. Ikada Y, Tabata Y. Protein release from gelatin matrices. Adv Drug Deliv Rev 1998;31:287-301.

12. Tabata Y, Nagano A, Ikada Y. Biodegradation of hydrogel carrier incorporating fibroblast growth factor. Tissue Eng 1999; 5:127-138.

13. Iwanaga K, Yabuta T, Kakemi M, Morimoto K, Tabata $Y$, Ikada Y. Usefulness of microspheres composed of gelatin with various cross-linking density. J Microencapsul 2003;20: 767-776.

14. Verma RP, Hansch C. Matrix metalloproteinases (MMPs): Chemical-biological functions and (Q)SARs. Bioorg Med Chem 2007;15:2223-2268.

15. Holland TA, Tabata Y, Mikos AG. Dual growth factor delivery from degradable oligo(poly(ethylene glycol) fumarate) hydrogel scaffolds for cartilage tissue engineering. J Control Release 2005;101:111-125.

16. Fraker PJ, Speck JC Jr. Protein and cell membrane iodinations with a sparingly soluble chloroamide, 1,3,4,6-tetrachloro-3a, 6a-diphrenylglycoluril. Biochem Biophys Res Commun 1978; 80:849-857.

17. Habraken WJEM, de Jonge LT, Wolke JGC, Yubao L, Mikos AG, Jansen JA. Introduction of gelatin microspheres into an injectable calcium phosphate cement. J Biomed Mater Res A 2008;87:643-655.

18. Bigi A, Boanini E, Panzavolta S, Roveri N, Rubini K. Bonelike apatite growth on hydroxyapatite-gelatin sponges from simulated body fluid. J Biomed Mater Res 2002;59:709715 .

19. Blom EJ, Klein-Nulend J, Wolke JG, Kurashina K, van Waas MA, Burger EH. Transforming growth factor-beta1 incorporation in an alpha-tricalcium phosphate/dicalcium phosphate dihydrate/tetracalcium phosphate monoxide cement: Release characteristics and physicochemical properties. Biomaterials 2002;23:1261-1268.

20. Ruhe PQ, Kroese-Deutman HC, Wolke JG, Spauwen PH, Jansen JA. Bone inductive properties of rhBMP-2 loaded porous calcium phosphate cement implants in cranial defects in rabbits. Biomaterials 2004;25:2123-2132.

21. del Real RP, Ooms E, Wolke JG, Vallet-Regi M, Jansen JA. In vivo bone response to porous calcium phosphate cement. J Biomed Mater Res A 2003;65:30-36.

22. Ooms EM, Wolke JGC, van den Heuvel MT, Jansen JA. Histological evaluation of the bone response to calciumphosphate cement implanted in cortical bone. Biomaterials 2003; 24:989-1000.

23. Dhert WJ, Klein CP, Wolke JG, van der Velde EA, de Groot $\mathrm{K}$, Rozing PM. A mechanical investigation of fluorapatite, magnesiumwhitlockite, and hydroxylapatite plasma-sprayed coatings in goats. J Biomed Mater Res 1991;25:1183-1200. 